\title{
A novel cationic nanostructured lipid carrier for improvement of ocular bioavailability: design, optimization, in vitro and in vivo evaluation
}

Rui Liu ${ }^{\text {a, }}$, Shuangshuang Wang ${ }^{\text {a }}$, Lu Sun ${ }^{\text {a }}$, Shiming Fang ${ }^{\text {b }}$, Jialu Wang a , Xingguo Huang a, Zhen You ${ }^{a}$, Xin $\mathrm{He}^{\mathrm{a}, \mathrm{b}}$, Changxiao Liu ${ }^{\mathrm{c}}{ }^{*}$

a School of Chinese Materia Medica, Tianjin University of Traditional Chinese Medicine, 312 Anshanwest Road, Nankai District, Tianjin, 300193, China

b Tianjin State Key Laboratory of Modern Chinese Medicine, 312 Anshanwest Road, Nankai District, Tianjin, 300193 , China

c State Key Laboratory of Drug Delivery Technology and Pharmacokinetics, Tianjin Institute of Pharmaceutical Research, 308 Anshanwest Road, Nankai District, Tianjin, 300193, China

Address for correspondence: Rui Liu, School of Traditional Chinese Materia Medica, Tianjin University of Traditional Chinese Medicine, Tianjin 300193, China. Tel.: +86-22-59596221; fax: +86-22-59596221; E-mail: 1r_8000@163.com. Changxiao Liu, State Key Laboratory of Drug Delivery Technology and Pharmacokinetics, Tianjin Institute of Pharmaceutical Research, Tianjin 300193, China. Tel.: +86-22-84845243; fax: +86-22-84845243; E-mail: liuchangxiao@163.com. 


\section{ABSTRACT}

The objective of this study was to prepare, optimize and evaluate a novel cationic nanostructured lipid carrier (CNLC) system, which can enhance the ophthalmic bioavailability of curcumin (CUR). CUR-loaded CNLC (CUR-CNLC) was prepared using a film-ultrasonic technique. A three-level Box-Behnken design was applied for optimization. The physicochemical properties, in vitro release and corneal permeation of the delivery method were evaluated. Preocular retention capacity study was conducted using a non-invasive fluorescence imaging system. Finally, a pharmacokinetic study in the aqueous humor was performed using a microdialysis technique. The optimal formulation had a mean particle size of $158.1 \mathrm{~nm}$ with a homogeneous distribution, a positive surface charge of 36.5 $\mathrm{mV}$, a high entrapment efficiency of $99.12 \%$ and drug loading of $1.677 \%$. Transmission electron microscope (TEM) morphology depicted a spherical morphology, and differential scanning calorimetry (DSC) indicated an imperfect crystalline lattice for the formulation. The CUR-CNLC formulation exhibited a sustained drug release and a 1.25 -fold increase in in vitro corneal permeation $(P<0.05)$. The results from in vivo studies indicated the formulation had a high tolerance and a prolonged drug retention capacity in ocular tissues. A pharmacokinetic study of rabbit aqueous humors demonstrated a 2.36 -fold increase in ocular bioavailability compared to a CUR solution (CUR-SOL, $P<0.01$ ). The amphipathic octadecyl-quaternized carboxymethyl chitosan (QACMC) was used as a novel cationic material to prolong the residence time and improve the ocular bioavailability. Therefore, CNLC system is a promising approach for ocular delivery of CUR.

\section{Keywords}


cationic nanostructured lipid carriers; microdialysis; pharmacokinetics; amphipathic octadecyl-quaternized carboxymethyl chitosan; ocular delivery; curcumin

\section{Introduction}

The topical instillation of an active compound is the first-choice delivery method for ocular therapy. However, the physiological constraints imposed by the protective mechanisms of the eye lead to low absorption of drugs and result in a short duration of therapeutic effect [1]. When drug solution is dropped into the eye, the effective tear drainage and blinking action cause a 10-fold reduction in the concentration of the drug within 4-20 min [2,3]. Most of the administered dose passes through the naso-lacrimal duct into the gastrointestinal tract, which may cause side effects [4]. In addition, the limited permeability of the cornea also contributes to the poor absorption of ocular drugs [1].

Nanostructured lipid carrier (NLC) systems are second generation lipid-based nanoparticles obtained by substituting part of the solid lipids used in solid lipid nanoparticles (SLN) formulations with liquid lipids. Compared with other traditional carriers, NLC have many excellent characteristics, including high bioavailability, high active ingredient payload, biodegradability, good tolerance, prevention or minimization of active ingredient expulsion, avoidance of organic solvent use during preparation, improvement of physicochemical stability, and suitability for large-scale production using existing technologies and sterilization [5]. A cationic nanostructured lipid carrier (CNLC) system combines the positive surface charge of the particles and the negative surface charge of the ocular mucosa by means of electrostatic attraction [6]. Cationic materials are able to improve the bio-adhesion of NLC and prolong the retention time of drugs in the eyes. The biodegradability of the cationic materials is a basic requirement to avoid their accumulation in the ocular tissues because various clinical applications using drug delivery to the eye 
require chronic administration. Chitosan, known as a biodegradable and biocompatible polymer, is suitable for delivery via mucosal routes. Early studies by Baldrick [7] and Fulgêncio [8] presented chitosan as a promising cationic biomaterial with excellent biocompatibility for both ophthalmic and intravenous administration. In addition, a number of studies both in vitro and in vivo showed great biocompatibility and tolerance when using chitosan-based nanoparticles [9-11].

Curcumin (CUR) is isolated from the rhizomes of Curcuma [12]. A growing body of experimental evidence suggests the various beneficial pharmacological effects of CUR, including its anti-oxidant, anti-inflammatory, anti-carcinogenic, anti-bacterial, and anti-coagulant features [13]. CUR capsules are useful for diabetic patients, aiding them in obtaining better control over blood glucose and decreasing the incidence of eye disease [14]. CUR has been proven to be effective for ophthalmic use in various ocular fundus pathologies. It inhibits the proliferation of human lens epithelial cells and protects retinal cells, retinal ganglion cells, and corneal epithelial cells $[15,16]$. However, owing to drawbacks such as low solubility and bioavailability, the clinical application of CUR is limited.

The response surface methodology (RSM) is useful in simultaneously analyzing process variables when variable interactions are very complicated. Box-Behnken design is one of the techniques in RSM. It is a well-established approach for pharmaceutical formulation development and optimization, allowing the extraction of maximal information out of a few well-designed experiments $[17,18]$. This design is a convenient approach for experimental optimization, which has a large number of variables. It is more efficient and economical than other designs [19].

Therefore, it is necessary to develop a novel ophthalmic drug delivery system not only to increase the aqueous solubility of CUR but also to overcome the shortcomings of conventional dosage forms. The objective of the present study was to prepare, optimize and 
evaluate a novel CNLC system to enhance the ophthalmic bioavailability of CUR. A CNLC formulation was developed using the film-ultrasonic method. A well-established three-level Box-Behnken design was used to optimize the CUR-CNLC formulation. The CUR-CNLC formulation was further characterized for particle size, zeta potential, morphology and crystallinity. In vitro drug release testing was conducted, and corneal permeation was performed using excised rabbit cornea. Additionally, in vivo tolerability and retention capability were assessed with ocular irritation and preocular retention time studies. Finally, ocular bioavailability was evaluated using the microdialysis technique. To our knowledge, this is the first integrated report on the enhancement of the ocular bioavailability of CUR following the topical administration of a CNLC delivery system.

\section{Materials and methods}

\subsection{Materials}

CUR was purchased from Zelang Medical Technology Co., Ltd. (98\%, Nanjing, Jiangsu, China). Glycerin monostearate (GMS), Tween-80, stearic acid and tristearin were provided by the Tianjin Chemical Reagent Company (Tianjin, China). Compritol® 888 ATO, Labrasol ${ }^{\circledR}$, Gelucire ${ }^{\circledR} 44 / 14$, Transcutol ${ }^{\circledR}$ P and Transcutol ${ }^{\circledR}$ HP were gifts from Gattefosse S.A. (Saint-Priest, France). Miglyol® 812, Miglyol ${ }^{\circledR}$ 840, Myri 52 and Super Refined ${ }^{\circledR}$ Oleic Acid were generously provided by Fengli Jingqiu Commerce and Trade Co., Ltd. (Beijing, China). Hydroxypropyl chitosan, carboxymethyl chitosan, and amphipathic octadecyl-quaternized carboxymethyl chitosan (QACMC) were supplied by Nantong Lushen Biological Engineering Co., Ltd. (Nantong, Jiangsu, China). All other reagents were of analytical grade.

\subsection{Production of CUR-CNLC}


In the present work, two chemically different lipids (GMS and Miglyol ${ }^{\circledR}$ 812) were selected from a list of solid and liquid lipids. The selection rationale was to choose lipids that could better dissolve CUR and produce CUR-CNLC. Myri 52 was chosen as a surfactant. QACMC was used as a cationic material. CUR-CNLC was prepared using a film-ultrasonic technique. Briefly, ethanol was added to the lipid phase containing $2 \mathrm{mg}$ CUR, $25.45 \mathrm{mg}$ GMS and $14.55 \mathrm{mg}$ Miglyol ${ }^{\circledR} 812$. Then, the lipid phase was heated to $80{ }^{\circ} \mathrm{C}$ to melt the lipids. Subsequently, a rotary evaporator (RE-52AA, Ya Rong Biochemical Instrument Co., Ltd., Shanghai, China) was used to remove ethanol in the lipid phase at $80{ }^{\circ} \mathrm{C}$. Meanwhile, $50.10 \mathrm{mg}$ Myri 52, $21.21 \mathrm{mg}$ Gelucire ${ }^{\circledR} 44 / 14$ and $10.00 \mathrm{mg}$ QACMC were added to $50 \mathrm{~mL}$ of distilled water heated to $80^{\circ} \mathrm{C}$. The solution was stirred using a magnetic heated stir plate (SZCL-4B, Yu Hua Instrument Co., Ltd., Henan, China) to obtain a homogeneous lipid solution. A small amount of ethanol was added to the hot lipid phase and then it was dispersed in the aqueous phase to form a pre-emulsion. After stirring at $80{ }^{\circ} \mathrm{C}$ for $0.5 \mathrm{~h}$, the pre-emulsion was treated with an ultrasonic cleaner (KH 2200B, He Chuang ultrasonic Co., Ltd., Shanghai, China) at the same temperature for $0.5 \mathrm{~h}$. The resulting hot $\mathrm{O} / \mathrm{W}$ nano-emulsion was cooled to $0{ }^{\circ} \mathrm{C}$ and then restirred to form CUR-CNLC. CUR-SOL was prepared by adding the same quantity of CUR with the optimal CUR-CNLC formulation and appropriate amount of Tween- 80 into $50 \mathrm{~mL}$ distilled water. CUR-NLC was produced using the same formulation without QACMC and employed for comparison against CUR-CNLC and CUR-SOL in in vitro and in vivo studies.

\subsection{Drug analysis}

The quantitative determination of CUR was performed using a Waters e2695 high-performance liquid chromatography (HPLC) system (Waters Ltd., Milford, U.S.). The detector was set at $425 \mathrm{~nm}$. A reverse-phase column (Diamonsil C18 (2), $5 \mu \mathrm{m}, 200 \times 4.6 \mathrm{~mm}$ ) 
was used, and the column temperature was set at $35{ }^{\circ} \mathrm{C}$. The mobile phase consisted of methanol and $0.05 \%(\mathrm{v} / \mathrm{v})$ methanoic acid $(80: 20)$. The flow rate of the mobile phase was 1 $\mathrm{mL} \cdot \min ^{-1}$

\subsection{Optimization design of CUR-CNLC}

During the preliminary study, the influence of each parameter on the physicochemical properties of CUR-CNLC was assessed. To assess the optimal condition of the technical procedure, a four-factor, three-level Box-Behnken design set up by Design-Expert 8.06 (Stat Ease, Inc., Minneapolis, U.S.) was undertaken as described in Table 1. The independent variables were dosage of lipid $\left(\mathrm{X}_{1}\right)$, the ratio between solid lipid and liquid lipid $\left(\mathrm{X}_{2}\right)$, dosage of surfactant $\left(\mathrm{X}_{3}\right)$ and dosage of cationic material $\left(\mathrm{X}_{4}\right)$. The established dependent variables were the geometric mean (OD), which can be calculated by the following formulas 1 and 2:

$$
\begin{gathered}
d_{n}=\frac{Y_{i}-Y_{\text {min }}}{Y_{\text {max }}-Y_{\text {min }}} \\
O D=\left(d_{1} d_{2} \cdots d_{n}\right)^{\frac{1}{n}}
\end{gathered}
$$

where $Y_{i}$ is the observed values of drug loading (DL), entrapment efficiency (EE) and appearance score of CUR-CNLC; $Y_{\max }$ and $\mathrm{Y}_{\min }$ are the acceptable maximal and minimal values of the response variable, respectively; and $\mathrm{n}$ is the number of response variables [20, 21].

\subsection{Entrapment efficiency and drug loading}

The EE and DL of CUR in CUR-CNLC was assessed indirectly. Non-entrapped CUR was removed with a filtration-centrifugation technique using UFC 510024 centrifugal filter devices (Amicon ${ }^{\circledR}$ Millipore Corporation, Bedford, MA, U.S.) at $8000 \mathrm{rpm}$ for $10 \mathrm{~min}$ [22].

\subsection{Mean particle size and Zeta potential analysis}


Mean particle size (PS), polydispersity index (PI) and zeta potential (ZP) were determined by a Zetasizer Nano ZS (Malvern Instruments Ltd., Worcestershire, UK) after appropriate dilution with ultra-purified water at $25^{\circ} \mathrm{C}$.

\subsection{Differential scanning calorimetry}

DSC analysis was performed using a Mettler Toledo DSC (1/500, Mettler-Toledo Ltd., Shanghai, China) equipped with a refrigerated cooling system. Bulk materials, a physical mixture of bulk materials, CUR and a freeze-dried powder of CUR-CNLC were put into aluminum pans. Their thermal analysis profiles were obtained as the temperature increased from $-40{ }^{\circ} \mathrm{C}$ to $220{ }^{\circ} \mathrm{C}$ at a scanning rate of $5{ }^{\circ} \mathrm{C} \cdot \mathrm{min}^{-1}$ under a nitrogen atmosphere. An empty pan was used as a reference.

\subsection{Morphological study}

The optimized CUR-CNLC formulation was examined morphologically in a TEM (HT7700, Hitachi, Tokyo, Japan). After diluting the sample 50-fold with double distilled water, a drop of the sample was immediately spread on a film-coated copper grid, and the excess liquid was absorbed by filter paper. Then, the sample was dried at ambient atmosphere.

\subsection{In vitro drug release test}

In vitro release of CUR from CUR-CNLC was carried out with a dialysis membrane method [23]. A total of $2 \mathrm{~mL}$ of CUR-SOL, CUR-NLC and CUR-CNLC were put in dialysis bags with a molecular weight cut off of $8000-14,000$. The release medium was $50 \mathrm{~mL}$ of $40 \%$ ethanol $(\mathrm{v} / \mathrm{v}, \mathrm{pH}=4.0)$ at $34 \pm 1{ }^{\circ} \mathrm{C}$ [24]. The dialysis bags were suspended in release medium on a magnetic heated stir plate and stirred at $150 \mathrm{rpm}$. A total of $1 \mathrm{~mL}$ of sample was withdrawn at predetermined time intervals and replaced with fresh medium immediately for 
$12 \mathrm{~h}$. Finally, the samples were analyzed by RP-HPLC. The experiments were conducted in three repeats.

\subsection{In vitro corneal permeation evaluation and hydration level}

The in vitro corneal permeation profiles of CUR-SOL, CUR-NLC and CUR-CNLC formulations were evaluated using excised corneas of rabbits according to the previous method by Hao [25]. Freshly excised rabbit corneas were immediately mounted on Franz-type diffusion cells, which were maintained at a constant temperature $34 \pm 1{ }^{\circ} \mathrm{C}$, under stirring conditions using a transdermal diffusion machine at a rotating speed of $200 \mathrm{rpm}$ (YB-P6, Tianjin, China). Each formulation $(500 \mu \mathrm{L})$ was added to the donor chamber, and $4.5 \mathrm{~mL}$ fresh glutathione bicarbonate Ringer (GBR) buffer with 3\% Tween-80 (v/v) was placed in the reception cell. GBR solution is reported to preserve tissue integrity of excised corneas over $6 \mathrm{~h}$ [26]. Tween-80 was used as a solubilizer to increase the solubility of curcumin. At regular time intervals $(40,80,120,160,200$ and $240 \mathrm{~min})$, samples $(4.5 \mathrm{~mL})$ from the receptor chamber were withdrawn and replaced with an equal volume of fresh GBR. At the end of the corneal permeation experiment, each cornea was desiccated at $60^{\circ} \mathrm{C}$ for $16 \mathrm{~h}$ and weighed to calculate the corneal hydration level. Each experiment was performed in triplicate.

\subsection{In vivo study}

\subsubsection{Animals}

New Zealand white rabbits, with weight of $2.5-3.0 \mathrm{~kg}$, were used in the present study. The animals were maintained at $25 \pm 1{ }^{\circ} \mathrm{C}$ and $50 \pm 5 \% \mathrm{RH}$ in a light-controlled room with $\mathrm{ad}$ libitum acess to food and water. The procedures involving animals were reviewed and approved by the Animal Ethical Committee at Tianjin University of Traditional Chinese 
Medicine.

\subsubsection{Ocular irritation study}

An ocular irritation study was performed with six rabbits according to a modified Draize technique [27]. CUR-NLC and CUR-CNLC formulations were instilled into the left eyes, and PBS (pH 7.4) was instilled into the right eyes at a rate of $0.1 \mathrm{~mL}$ every $4 \mathrm{~h}$, four times a day for a period of 7 days. The condition of the ocular tissue was monitored at 4, 12, 24, 48, and $72 \mathrm{~h}$ after the end of the last instillation.

\subsubsection{Preocular retention time study}

The preocular retention of CUR-CNLC was assessed using a non-invasive fluorescence imaging system. CUR was replaced with Rh B in the oil phase and was then processed via the same method as for the preparation of CNLC. The albino rabbits were maintained in a sober state. Just before the time of imaging, they were anesthetized with chloral hydrate (injection $2.5 \mathrm{ml} \cdot \mathrm{kg}^{-1}$ ) by ear vein. Exactly $20 \mu \mathrm{L}$ of the formulation was instilled directly into the conjunctival sac of the right eye, and the left eye was left as a control group. The eyes were manually closed for $10 \mathrm{~min}$ to allow distribution over the cornea. At $0,15,30,60$, 90, 120 and $150 \mathrm{~min}$, imaging was carried out. The region of interest (ROI) for the residual fluorescence was around the ocular and non-ocular areas $[28,29]$. The remaining intensity $(\mathrm{R})$ was calculated according to the following formula 3 :

$$
\mathrm{R} \%=\frac{\mathrm{A}-\mathrm{B}}{\mathrm{C}} \times 100
$$

where A was the intensity of ROI, B was the background fluorescence intensity, and C was the intensity of the ROI at 0 min [28].

\subsubsection{Pharmacokinetic study in aqueous humor}


A pharmacokinetic study in the aqueous humor was carried out using microdialysis. The experimental operation was performed as previously reported by Liu [30]. Briefly, three rabbits were locally anesthetized with $20 \mathrm{mg} \cdot \mathrm{kg}^{-1}$ of lidocaine hydrochloride injection. A microdialysis probe (MD 2000, Bioanalytical Systems, Inc, West Lafayette, Indiana) was implanted into the aqueous humor using a $20 \mathrm{G}$ needle. The needle was removed, leaving the probe with the microdialysis membrane in the middle of the anterior chamber. The probe was perfused with GBR containing $0.06 \%$ Tween- 80 at a flow rate of $3 \mu \mathrm{L} \cdot \mathrm{min}^{-1}$ using a microdialysis pump (CMA106, CMA Microdialysis Co., Ltd, Stockholm, Sweden). The corneal wound surfaces were treated with $0.3 \%(\mathrm{w} / \mathrm{v})$ ofloxacin ophthalmic solution. Animals were allowed to stabilize for 1 day before topical drug administration.

Probe recovery was evaluated using a retrodialysis method. Different concentrations of standard CUR solutions were perfused, and dialysates were collected $15 \mathrm{~min}$ after $30 \mathrm{~min}$ of perfusion. In vivo recovery $(R)$ was calculated by the following formula 4 :

$$
R \%=\frac{C_{d}-C_{p}}{C_{m}-C_{p}} \times 100
$$

where $C_{d}, C_{p}$ and $C_{m}$ are the concentrations of the drug in the dialysate, perfusate and aqueous humor, respectively. $R$ is the value of the slope for the plot of $C_{d}-C_{p}$ versus $C_{p}$.

The CUR-SOL, CUR-NLC and CUR-CNLC formulations $\left(0.2 \mathrm{~mL}, 1.0 \mathrm{mg} \cdot \mathrm{mL}^{-1}\right)$ were instilled into different groups of rabbit eyes. Dialysates were collected every $20 \mathrm{~min}$ for the first $2 \mathrm{~h}$ and every $30 \mathrm{~min}$ thereafter. The study was repeated for three times, and all samples were analyzed using HPLC.

\subsection{Statistical analysis}

The optimization design of CUR-CNLC and the data were analyzed by Design-Expert software (8.06 version, Stat Ease, Inc., Minneapolis, U.S.) [31]. The results were presented as the mean \pm standard deviation (SD). Statistical analysis of the results was performed by 
two-way analysis of variance (ANOVA) and then one-way ANOVA using SPSS software

(17.0 version, Spss Inc., Illinois, U.S.). The statistical significance was set at $p<0.05$ [32].

\section{Results}

\subsection{Optimization and validation}

The results from preliminary studies indicated that the optimal parameters of the CUR-CNLC formulation are $2 \mathrm{mg}$ CUR, $60 \mathrm{mg}$ total liquid (30 mg GMS, $30 \mathrm{mg}$ Miglyol® 812), $70 \mathrm{mg}$ Myri 52, $20 \mathrm{mg}$ Gelucire ${ }^{\circledR}$ 44/14, $30 \mathrm{mg}$ cationic material and $50 \mathrm{~mL}$ water. Each experimental response could be represented by the following formula:

$$
\begin{aligned}
& \mathrm{Y}=\mathrm{aX}+\mathrm{bX}_{2}+\mathrm{c} \mathrm{X}_{3}+\mathrm{dX}_{4}+\mathrm{eX}_{1} \mathrm{X}_{2}+\mathrm{fX}_{1} \mathrm{X}_{3}+\mathrm{gX}_{1} \mathrm{X}_{4}+\mathrm{hX} \mathrm{X}_{3}+\mathrm{iX}_{2} \mathrm{X}_{4}+\mathrm{jX}_{3} \mathrm{X}_{4}+\mathrm{kX}_{1}^{2}+\mathrm{lX}_{2}^{2}+\mathrm{mX}_{3}^{2}+\mathrm{nX}_{4}^{2} \\
& +\mathrm{o} \mathrm{X}_{1}^{2} \mathrm{X}_{2}+\mathrm{pX}_{1}^{2} \mathrm{X}_{3}+\mathrm{q} \mathrm{X}_{1}^{2} \mathrm{X}_{4}+\mathrm{rX}_{1} \mathrm{X}_{2}^{2}+\mathrm{sX}_{1} \mathrm{X}_{3}^{2}+\mathrm{tX}_{2}^{2} \mathrm{X}_{3}+\mathrm{uX}_{2}^{2} \mathrm{X}_{4}+\mathrm{v} \mathrm{X}_{2} \mathrm{X}_{3}^{2}+\mathrm{w}
\end{aligned}
$$

where $\mathrm{Y}$ is the response, $\mathrm{X}_{1}$ is the concentration of lipid, $\mathrm{X}_{2}$ is the ratio between solid lipid and liquid lipid, $\mathrm{X}_{3}$ is the concentration of surfactant, $\mathrm{X}_{4}$ is the concentration of cationic material, $\mathrm{w}$ is a constant, a to $\mathrm{v}$ are the regression coefficients.

The responses of optimization experiments can be modeled by the two-factor model, the quadratic model and the adjusted cubic model are shown in Table 2. It was demonstrated that the best-fit model for the responses was the adjusted cubic model, which had the maximal $r$ value. ANOVA was carried out to evaluate the significance of the cubic model terms on response and their quantitative effects. Through comparing the associated p-value with the F-value of each term at a $95 \%$ confidence level, the appropriate model terms were aliased. Compared to the two-factor model and the quadratic model, the adjusted cubic model had the largest $r$ value for the response. The adjusted cubic model was chosen to describe the effects of the variables thereafter.

The sign and value of the quantitative effect represented the tendency and magnitude of the responses, respectively. EE, DL and the appearance score of CUR-CNLC were calculated 
to obtain the OD value and the OD value was input for the construction of RSM. The response surface analyses were also plotted in three-dimensional model graphs. The three-dimensional response surface plots for the OD values are presented in Figure 1. By analyzing these graphs, the optimal parameters of the CUR-CNLC formulation were determined to be $2 \mathrm{mg}$ CUR, $40 \mathrm{mg}$ total liquid (36.36\% GMS Miglyol® 812), $50.10 \mathrm{mg}$ Myri 52, $21.21 \mathrm{mg}$ Gelucire ${ }^{\circledR}$ 44/14 (0.04\% wt), $10 \mathrm{mg}$ QACMC and $50 \mathrm{~mL}$ water. The EE and DL of the optimal formulation were $99.12 \pm 0.01 \%$ and $1.677 \pm 0.19 \%$, respectively. This formulation was considered to be the most appropriate formulation for ophthalmic application and was employed for further characterization.

\subsection{Physicochemical characterization}

Figure $2 \mathrm{a}$ and $2 \mathrm{~b}$ depict the unimodal distribution profiles of PS and ZP. The PS of the optimized formulation was $158.1 \mathrm{~nm}$, the PI was 0.29 , and the $\mathrm{ZP}$ was $36.5 \mathrm{mV}$.

\subsection{Differential scanning calorimetry}

The drug entrapment was further characterized by DSC to reveal the state of the encapsulated drug. Figure 3 shows DSC thermograms of QACMC, Myri 52, Miglyol ${ }^{\circledR} 812$, GMS, CUR, a physical mixture of the bulk materials of the optimal CUR-CNLC formulation and a freeze-dried powder of the optimal CUR-CNLC formulation. The thermogram of the bulk materials physical mixture indicated a melting process of Myri 52, Miglyol $\mathbb{R} 812$ and GMS with a maximal tri-peak at $-3-64{ }^{\circ} \mathrm{C}$. The peak of QACMC was at $119{ }^{\circ} \mathrm{C}$. The DSC curve of CUR showed a melting endotherm at $178.8{ }^{\circ} \mathrm{C}$, whereas it was not observed for CUR-CNLC. This could be due to the presence of CUR in a dissolved state in the lipid matrix [33]. The results illustrated that the CUR in CUR-CNLC was in an amorphous state 
[34].

\subsection{Morphological study}

The TEM image in Figure 4 reveals that the optimized CUR-CNLC formulation was dispersed as individual CNLC with a well-defined spherical shape and a homogeneous distribution of approximately $160 \mathrm{~nm}$ in diameter.

\subsection{In vitro drug release test}

The in vitro release profiles of CUR from CUR-SOL, CUR-NLC and CUR-CNLC are displayed in Figure 5. It was observed that 90.37\% CUR was released from CUR-SOL in 12 h. CUR-CNLC showed a biphase release pattern: a small initial burst release $(19.73 \%)$ over a period of $20 \mathrm{~min}$, followed by a sustained release (80.94\%) over a period of $12 \mathrm{~h}$. The in vitro release data were fitted to different kinetic equations, including zero order, first order, Higuchi, Hixcon-Crowell, Ritger-Peppas and Weibull models. The fitted equations are shown in Table 3. The best fit kinetic model was the Weibull model ( $\mathrm{r}=0.9933)$. Compared with CUR-NLC, CUR-CNLC displayed a more sustained release phase in the release profiles. This sustained release mainly resulted from the erosion and degradation of the components of CUR-CNLC [35].

\subsection{In vitro corneal permeation evaluation}

Table 4 lists the corneal permeability coefficients and HL values determined for the CUR-SOL, CUR-NLC and CUR-CNLC formulations. The CUR-NLC and CUR-CNLC formulations showed $P_{a p p}$ values higher than those of CUR-SOL. CUR-NLC and CUR-CNLC exhibited a remarkable respective 1.24-fold and 1.25-fold increase compared with that of CUR-SOL $(P<0.05)$. The HL values indicated that the three formulations did not cause any damage to the epithelium or endothelium during this study. 


\subsection{In vivo study}

\subsubsection{Ocular irritation study}

The ocular irritation scores of both CUR-NLC and CUR-CNLC were $2 \pm 0$. No signs of toxicity or irritation to the external ocular tissues were observed after the Draize test [27]. Although there was a slight hyperemia in the conjunctiva, no ocular damage or abnormal clinical signs in the cornea or iris were visible.

\subsubsection{Preocular retention time study}

As shown in Figure 6, rapid clearance could be observed in the Rh B solution group after administration. Fluorescence intensity remaining in the ROI at $30 \mathrm{~min}$ after administration was defined as $\mathrm{A}_{30}$. At $30 \mathrm{~min}$, only $37.44 \%$ fluorescence intensity remained in the ROI, which was significantly lower than that of Rh B-CNLC, $\mathrm{A}_{30}$ of Rh B-CNLC was $51.36 \%$, as displayed in Figure 7. At 90 min, the statistical analysis noted that $24.52 \%$ and $31.87 \%$ intensity remained in the $\mathrm{Rh} \mathrm{B}$ solution and $\mathrm{Rh} \mathrm{B}-\mathrm{CNLC}$ group, respectively. This indicated that the CNLC formulation exhibited a relatively strong intensity and slow clearance from the ROI.

\subsubsection{Pharmacokinetic study in aqueous humor}

In vivo probe recovery was determined before the pharmacokinetic study to ensure that the implanted probes were in good function. The in vivo recovery was $50.75 \pm 3.91 \%(\mathrm{n}=3)$.

Aqueous humor pharmacokinetics parameters are summarized in Table 5. As shown in Figure 8, compared with CUR-SOL, the area under the concentration-time curve (AUC) value was enhanced by 2.36 -fold for CUR-CNLC $(p<0.01)$, and concentration at peak $\left(\mathrm{C}_{\max }\right)$ value of CUR-CNLC was enhanced by 1.28 -fold $(p<0.01)$. The time to peak concentration ( $\mathrm{T}_{\max }$ ) of CUR-CNLC was determined to be up to $126.7 \mathrm{~min}$, and the MRT value was 
extended. There were significant differences in the $\mathrm{T}_{\max }$ and MRT values of CUR-CNLC compared with those of CUR-SOL $(p<0.01)$.

\section{Discussion}

\subsection{Selection of materials and preparation methods}

Due to the physiological constraints imposed by the protective mechanisms of the eye, a nanotechnology-based drug delivery system was chosen to improve the bioavailability of CUR. In recent years, drug delivery systems of cationic nanoparticles have shown greater stability and prolonged circulation half-live over traditional drug delivery systems [36]. QACMC was chosen as a promising cationic material for ocular drug delivery for the following reasons. First, QACMC can improve the water solubility of lipid soluble drugs, as well as the EE and DL of the NLC due to its perfect amphiphilicity [37]. Second, steric stabilization of QACMC is able to protect the nanoparticles from undesired attacks in biological media, thereby increasing the stability of the nanoparticles and prolonging their circulation time [38]. Third, its cationic character can be employed as a target for ophthalmic drug delivery. Fourth, the viscosity of QACMC can prolong the retention of the drug and improve its bioavailability in ocular administration $[39,40]$.

To improve corneal permeability, an absorption enhancer was added to the CUR-CNLC system. Gelucire ${ }^{\circledR} 44 / 14$ has been reported to improve the drug bioavailability of poorly water-soluble drugs without causing tissue damage at low concentrations [41]. Therefore, Gelucire ${ }^{\circledR} 44 / 14$ was selected for inclusion in the CUR-CNLC formulation as an absorption enhancer to improve ocular drug permeation.

Different techniques can be employed to prepare NLC, including ultrasonication, homogenization, melt-emulsification and low-temperature solidification methods [42]. Film ultrasonic processing does not involve large amounts of surfactants and organic solvents, 
which must be avoided in ophthalmic formulations. Therefore, it is considered to be an appropriate technique for the preparation of CNLC for ocular application.

\subsection{Characterization of CUR-CNLC}

Much of the published data has suggested that an appropriate particle size and a narrow size range ensure low irritation, adequate bioavailability and good compatibility with ocular tissues [43]. The type and amount of cationic modified material had immense effect on the particle size and its distribution. An increase in the concentrations of the cationic materials increased the viscosity of the aqueous phase, thus hindering the rapid dispersion of the cationic materials in the organic phase. Thus, a larger particle size was obtained for the formulations containing higher concentrations of cationic materials.

\subsection{In vitro study}

Drug release from CUR-CNLC involves multiphases. Burst effect was observed due to initial release of drug absorbed on the surface of nanoparticles [44]. On the one hand, the initial burst release is attributed to the large specific surface portion of the NLC from which the drug molecules desorbed. On the other hand, the sustained release pattern can be associated with the retention capacity of the lipophilic bilayers of the oily nanodroplets [45]. This factor is expected to be incorporated owing to its low aqueous solubility. Both initial burst release and sustained release are of interest for clinical applications. Initial burst is useful to improve drug permeation for a faster onset of action, while sustained release supplies drug over a prolonged period of time. In addition, the positive charge of CUR-CNLC can elicit electrostatic adhesion or interaction with the negatively charged mucin of the corneal epithelium, hence increasing ocular residence time on the corneal surface [46]. Thus, the corneal permeation of the drugs was also enhanced. 


\subsection{In vivo study}

$\mathrm{Rh} \mathrm{B}$ as a fluorescent dye was encapsulated in the nanoparticles, which can reflect retention capacity of LCNPs. Rh B has the characteristics of lipophilic. It may be well trapped into CNLC, so the nanoparticles are labeled by $\mathrm{Rh} B$ [28]. Results of the pharmacokeinetic study demonstrate that CUR-CNLC can prolong the retention of CUR following topical administration. The enhanced ocular bioavailability provided by the CNLC delivery system may be attributed to the following reasons. First, nanoparticles possess a bioadhesive property due to their extremely small particle size and increased surface area. Second, the encapsulation of CUR embedded into nanoparticles facilitates transport through the corneal route, leading to a maintained corneal gradient of drug concentration. Third, the cationic character of QACMC is able to prolong the retention of a drug and improve its bioavailability in ocular administration. Fourth, Gelucire ${ }^{\circledR} 44 / 14$ enhances the absorption of poorly soluble drugs, contributing to improved drug bioavailability [41]. Therefore, all of these factors ensure intimate contact with the epithelial mucosal surface of the eye, preventing tear wash out and consequently providing sustained drug release and a prolonged drug retention time.

\section{Conclusions}

A novel CUR-CNLC delivery system with the addition of the cationic material QACMC for ocular application was successfully developed and evaluated. The optimized formulation showed an appropriate particle size, a sustained release property, improved corneal permeability, high ophthalmic tolerability, and most importantly prolonged retention capacity. Therefore, CNLC system is a potential approach to improve ocular bioavailability and safe profile of poorly water-soluble drugs. 


\section{Acknowledgements}

We are thankful to Gattefoss é SAS (Saint-Priest, France), BASF (Ludwigshafen, German) and Beijing Fengli Jingqiu Commerce and Trade Co., Ltd (Beijing, China) for samples support of this work. This work was funded by National Natural Science Foundation of China (No. 81303142), Specialized Research Fund for the Doctoral Program of Higher Education of China (No. 20121210120001), Tianjin Research Program of Application Foundation and Advanced Technology (No. 14CQNJC13100), National Science Foundation for Post-doctoral Researchers (No. 2015M570231) and Program for Changjiang Scholars and Innovative Research Team in University (No. IRT_14R41).

\section{References}

[1] Z.D. Liu, X.H. Zhang, H.Y. Wu, et al., Preparation and evaluation of solid lipid nanoparticles of baicalin for ocular drug delivery system in vitro and in vivo, Drug Dev. Ind. Pharm. 37 (4) (2011) 475-481. doi: 10.3109/03639045.2010.522193.

[2] H. Gupta, M. Aqil, R. Khar, et al., Nanoparticles laden in situ gel of levofloxacin for enhanced ocular retention, Drug Deliv. 20 (7) (2013) 306-309. doi: 10.3109/10717544. 2013.838712 .

[3] L.H. Wang, X. Che, Y.X. Guo, et al., Thermoresponsive ophthalmic poloxamer/tween/ carbopol in situ gels of a poorly water-soluble drug fluconazole: preparation and in vitro-in vivo evaluation, Drug Dev. Ind. Pharm. 40 (10) (2015) 1402-1410. doi: 10.3109/ 03639045.2013 .828221$.

[4] D. Achouri, K. Alhanout, P. Piccerelle, et al., Recent advances in ocular drug delivery, Drug Dev. Ind. Pharm. 39 (11) (2013) 1599-1617. doi: 10.3109/03639045. 2012.736515. 
[5] J.M. Wang, J.G. Tang, X.F. Zhou, et al., Physicochemical characterization, identification and improved photo-stability of alpha-lipoic acid-loaded nanostructured lipid carrier, Drug Dev. Ind. Pharm. 40 (2) (2013) 201-210. doi: 10.3109/03639045. 2012.753901.

[6] F. Lallemand, P. Daull, S. Benita, et al., Successfully improving ocular drug delivery using the cationic nanoemulsion, novasorb, J. Drug Deliv. 2012 (2) (2012) 604204. doi: $10.1155 / 2012 / 604204$.

[7] P. Baldrick, The safety of chitosan as a pharmaceutical excipient, Regul. Toxicol. Pharmacol. 56 (3) (2010) 290-299. doi: 10.1016/j.yrtph.2009.09.015.

[8] G.O. Fulgêncio, F.A.B. Viana, R.R. Ribeiro, et al., New mucoadhesive chitosan film for ophthalmic drug delivery of timolol maleate: in vivo evaluation, J. Ocul. Pharmacol. Ther. 28 (4) (2012) 350-358. doi: 10.1089/jop.2011.0174.

[9] L. Contreras-Ruiz, M. de la Fuente, C. García-Vázquez, et al., Ocular tolerance to a topical formulation of hyaluronic acid and chitosan-based nanoparticles, Cornea 29 (5) (2010) 550-558. doi: 10.1097/ICO.0b013e3181bd9eee.

[10]M. De la Fuente, M. Raviña, P. Paolicelli, et al., Chitosan-based nanostructures: a delivery platform for ocular therapeutics, Adv. Drug Deliv. Rev. 62 (1) (2010) 100-117. doi: 10.1016/j.addr.2009.11.026.

[11]W. Tiyaboonchai, Chitosan nanoparticles: a promising system for drug delivery, Naresuan Univ. J. 11 (3) (2013) 51-66.

[12]M. Sun, X. Su, B.Y. Ding, et al., Advances in nanotechnology-based delivery systems for curcumin, Nanomedicine 7 (7) (2012) 1085-1100. doi: 10.2217/nnm.12.80.

[13] S. Dev, P. Prabhakaran, L. Filgueira, et al., Microfluidic fabrication of cationic curcumin nanoparticles as an anti-cancer agent, Nanoscale 4 (8) (2012) 2575-2579. doi: 10.1039/ c2nr11502f.

[14]P. Neerati, R. Devde, A.K. Gangi, Evaluation of the Effect of Curcumin Capsules on 
Glyburide Therapy in Patients with Type-2 Diabetes Mellitus, Phytother. Res. 28 (12) (2014) 1796-1800. doi: 10.1002/ptr.5201.

[15]B. Burugula, B.S. Ganesh, S.K. Chintala, Curcumin attenuates staurosporine-mediated death of retinal ganglion cells, Invest Ophthalmol Vis. Sci. 52 (7) (2011) 4263-4273. doi: 10.1167/iovs. 10-7103.

[16] Y.H. Hu, X.R. Huang, M.X. Qi, et al., Curcumin inhibits proliferation of human lens epithelial cells: a proteomic analysis, J. Zhejiang Univ. Sci. B 13 (5) (2012) 402-407. doi: 10.1631/jzus.B1100278.

[17]J.F. Hao, F.G. Wang, X.D. Wang, et al., Development and optimization of baicalinloaded solid lipid nanoparticles prepared by coacervation method using central composite design, Eur. J. Pharm. Sci. 47 (2) (2012) 497-505. doi: 10.1016/j.ejps. 2012.07.006.

[18]O.P. Ranjan, U.Y. Nayak, M.S. Reddy, et al., Optimization of Chronomodulated Delivery System Coated with a Blend of Ethyl Cellulose and Eudragit L100 by Central Composite Design: In Vitro and In Vivo Evaluation, J. Pharm. Innov. 9 (2) (2014) 95 105. doi: $10.1007 / \mathrm{s} 12247-014-9176-3$.

[19]J. Wu, H. Zhang, N.H. Oturan, et al., Application of response surface methodology to the removal of the antibiotic tetracycline by electrochemical process using carbon-felt cathode and DSA (Ti/RuO $\mathrm{RurO}_{2}$ ) anode, Chemosphere 87 (6) (2012) 614-620. doi: 10.1016/j.chemosphere.2012.01.036.

[20] Y.J. Jung, N.K.V. Truong, S. Shin, et al., A robust experimental design method to optimize formulations of retinol solid lipid nanoparticles, J. Microencapsul 30 (1) (2013) 1-9. doi: $10.3109 / 02652048.2012 .668958$.

[21]X.B. Yang, X.B. Wang, W.S. Pan, et al., Optimization and Characterization of Dry Powder of Fanhuncaoin for Inhalation Based on Selection of Excipients, Chem. Pharm. 
Bull. 59 (8) (2011) 929-937.

[22]J. Kawadkar, A. Pathak, R. Kishore, et al., Formulation, characterization and in vitro-in vivo evaluation of flurbiprofen-loaded nanostructured lipid carriers for transdermal delivery, Drug Dev. Ind. Pharm. 39 (4) (2013) 569-578. doi: 10.3109/ 03639045.2012.686509.

[23]J. Ezzati Nazhad Dolatabadi, H. Hamishehkar, H. Valizadeh, Development of dry powder inhaler formulation loaded with alendronate solid lipid nanoparticles: solid-state characterization and aerosol dispersion performance, Drug Dev. Ind. Pharm. 41 (9) (2015) 1431-1437. doi: 10.3109/03639045.2014.956111.

[24]J. Shaikha, D.D. Ankolab, V. Beniwal, et al., Nanoparticle encapsulation improves oral bioavailability of curcumin by at least 9-fold when compared to curcumin administered with piperine as absorption enhancer, Eur. J. Pharm. Biopharm. 37 (3-4) (2009) 223-230. doi: 10.1016/j.ejps.2009.02.019.

[25]J.F. Hao, X.D. Wang, Y.P. Bi, et al., Fabrication of a composite system combining solid lipid nanoparticles and thermosensitive hydrogel for challenging ophthalmic drug delivery, Colloids Surf. B Biointerfaces 114 (2014) 111-120. doi: 10.1016/j.colsurfb. 2013.09.059.

[26] W.J. O'Brien, H.F. Edelhauser, The corneal penetration of trifluorothymidine, adenine arabinoside, and idoxuridine: a comparative study, Investigative Ophthalmology \& Visual Science. 16 (12) (1977) 1093-1103.

[27]A. Czajkowska-Kosnik, E. Wolska, J. Chorazewicz, et al., Comparison of cytotoxicity in vitro and irritation in vivo for aqueous and oily solutions of surfactants, Drug Dev. Ind. Pharm. 41 (8) (2015) 1232-1236. doi: 10.3109/03639045.2014.938656.

[28]L. Gan, S. Han, J.Q. Shen, et al., Self-assembled liquid crystalline nanoparticles as a novel ophthalmic delivery system for dexamethasone: improving preocular retention and 
ocular bioavailability, Int. J. Pharm. 396 (1-2) (2010) 179-187. doi: 10.1016/j.ijpharm. 2010.06 .015

[29]H.Y. Wu, Z.D. Liu, J.J. Peng, et al., Design and evaluation of baicalin-containing in situ pH-triggered gelling system for sustained ophthalmic drug delivery, Int. J. Pharm. 410 (1-2) (2011) 31-40. doi: 10.1016/j.ijpharm.2011.03.007.

[30]R. Liu, Z.D. Liu, C.G. Zhang, et al., Nanostructured lipid carriers as novel ophthalmic delivery system for mangiferin: Improving in vivo ocular bioavailability, J. Pharm. Sci. 101 (10) (2012) 3833-3844. doi: 10.1002/jps.23251.

[31] S. Verma S, S.K. Singh, P.R.P. Verma, et al., Formulation by design of felodipine loaded liquid and solid self nanoemulsifying drug delivery systems using Box-Behnken design, Drug Dev. Ind. Pharm. 40 (10) (2014) 1358-1370. doi: 10.3109/03639045.2013. 819884.

[32]J. Varshosaz, M. Minayian, E. Moazen, Enhancement of oral bioavailability of pentoxifylline by solid lipid nanoparticles, J. Liposome Res. 20 (2) (2010) 115-123. doi: $10.3109 / 08982100903161456$.

[33]S. Bose S, B. Michniak-Kohn, Preparation and characterization of lipid based nanosystems for topical delivery of quercetin, Eur. J. Pharm. Sci. 48 (3) (2013) 442-452. doi: 10.1016/j.ejps.2012.12.005.

[34]J. Araujo, E. Gonzalez-Mira, M.A. Egea, et al., Optimization and physicochemical characterization of a triamcinolone acetonide-loaded NLC for ocular antiangiogenic applications, Int. J. Pharm. 393 (1-2) (2010) 167-175. doi: 10.1016/j.ijpharm.2010.03. 034.

[35]D.H. Liu, F.X. Liu, Z.H. Liu, et al., Tumor specific delivery and therapy by double-targeted nanostructured lipid carriers with anti-VEGFR-2 antibody, Mol. Pharm. 8 (6) (2011) 2291-2301. doi: 10.1021/mp200402e.

[36] M. Zhao, J. Chang, X.P. Fu, et al., Nano-sized cationic polymeric magnetic liposomes 
significantly improves drug delivery to the brain in rats, J. Drug Target 20 (5) (2012) 416-421. doi: 10.3109/1061186X.2011.651726.

[37]H.J. Wang, P.Q. Zhao, X.F. Liang, et al., Folate-PEG coated cationic modified chitosancholesterol liposomes for tumor-targeted drug delivery, Biomaterials 31 (14) (2010) 4129-4138. doi: 10.1016/j.biomaterials.2010.01.089.

[38] S. Parveen, R. Misra, S.K. Sahoo, Nanoparticles: a boon to drug delivery, therapeutics, diagnostics and imaging, Nanomedicine 8 (2) (2012) 147-166. doi: 10.1016/j.nano.2011. 05.016 .

[39] M. Larsson, W.C. Huang, M.H. Hsiao, et al., Biomedical applications and colloidal properties of amphiphilically modified chitosan hybrids, Prog. Polym. Sci. 38 (9) (2013) $1307-1328$.

[40]T.T. Ye, K. Yuan, W.J. Zhang, et al., Prodrugs incorporated into nanotechnology-based drug delivery systems for possible improvement in bioavailability of ocular drugs delivery, Asian J. Pharm. Sci. 8 (4) (2013) 207-217. doi:10.1016/j.ajps.2013.09.002.

[41]R. Liu, Z.D. Liu, C.G. Zhang, et al., Gelucire44/14 as a novel absorption enhancer for drugs with different hydrophilicities: in vitro and in vivo improvement on transcorneal permeation, J. Pharm. Sci. 100 (8) (2011) 3186-3195. doi: 10.1002/jps. 22540.

[42]D. Patel, S. Dasgupta, S. Dey, et al., Nanostructured lipid carriers (NLC)-based gel for the topical delivery of aceclofenac: preparation, characterization, and in vivo evaluation, Sci. Pharm. 80 (3) (2012) 749-764. doi: 10.3797/scipharm.1202-12.

[43]J. Singh, G. Chhabra, K. Pathak, Development of acetazolamide-loaded, pH-triggered polymeric nanoparticulate in situ gel for sustained ocular delivery: in vitro. ex vivo evaluation and pharmacodynamic study, Drug Dev. Ind. Pharm. 40 (9) (2014) 12231232. doi: 10.3109/03639045.2013.814061.

[44] V. Khurana, S. Patel, V. Agrahari, et al., Pazopanib pentablock coploymer nanoparticles 
for ocular neovascularization, Recent Patents on Nanomedicine 4 (1) (2014) 57-68. doi: $10.2174 / 1877912304999140930143244$.

[45] A. Seyfoddin, T. Sherwin, D.V. Patel, et al., Ex vivo and In vivo Evaluation of Chitosan Coated Nanostructured Lipid Carriers for Ocular Delivery of Acyclovir, Curr. Drug Deliv. Epub ahead of print. doi: 10.2174/1567201813666151116142752.

[46] R.M. Hathout, S. Mansour, N.D. Mortada, et al., Liposomes as an ocular delivery system for acetazolamide: in vitro and in vivo studies, AAPS PharmSciTech 8 (1) (2007) E1-E12. doi: 10.1208/pt0801001. 
Table 1. Initial 3-level design, providing the lower $(-1)$, medium $(0)$ and upper $(+1)$ level values for each variable.

\begin{tabular}{cccc}
\hline Variable & Low level (-1) & Medium level (0) & High level (1) \\
\hline $\mathrm{X}_{1} / \mathrm{mg}$ & 40 & 60 & 80 \\
$\mathrm{X}_{2}$ & $1: 4$ & $1: 1$ & $7: 4$ \\
$\mathrm{X}_{3} / \mathrm{mg}$ & 50 & 70 & 90 \\
$\mathrm{X}_{4} / \mathrm{mg}$ & 10 & 30 & 50 \\
\hline
\end{tabular}

$\mathrm{X}_{1}$, dosage of lipid; $\mathrm{X}_{2}$, the ratio between solid lipid and liquid lipid; $\mathrm{X}_{3}$, dosage of surfactant; $\mathrm{X}_{4}$, dosage of cationic material. 
Table 2. Coefficients of the models in optimization experiment.

\begin{tabular}{|c|c|c|c|}
\hline Coefficient & Two-factor model & Quadratic model & Adjusted cubic model \\
\hline $\mathrm{a}$ & $-2.6 \times 10^{-2}$ & $-4.1 \times 10^{-2}$ & $-1.7 \times 10^{-2}$ \\
\hline $\mathrm{b}$ & 0.28 & $3.3 \times 10^{-2}$ & -0.20 \\
\hline $\mathrm{c}$ & $-1.9 \times 10^{-2}$ & $-9.8 \times 10^{-3}$ & -0.10 \\
\hline $\mathrm{d}$ & $-5.2 \times 10^{-3}$ & $-2.1 \times 10^{-2}$ & -0.23 \\
\hline $\mathrm{e}$ & $3.6 \times 10^{-3}$ & $3.6 \times 10^{-3}$ & $5.5 \times 10^{-2}$ \\
\hline $\mathrm{f}$ & $2.9 \times 10^{-4}$ & $2.9 \times 10^{-4}$ & 0.12 \\
\hline $\mathrm{g}$ & $5.9 \times 10^{-5}$ & $5.9 \times 10^{-5}$ & $2.4 \times 10^{-2}$ \\
\hline $\mathrm{h}$ & $-4.9 \times 10^{-3}$ & $-4.9 \times 10^{-3}$ & $-7.4 \times 10^{-2}$ \\
\hline $\mathrm{i}$ & $-8.7 \times 10^{-3}$ & $-8.7 \times 10^{-3}$ & -0.13 \\
\hline $\mathrm{j}$ & $-4.3 \times 10^{-7}$ & $-4.3 \times 10^{-7}$ & $-1.7 \times 10^{-4}$ \\
\hline $\mathrm{k}$ & - & $1.3 \times 10^{-4}$ & $5.1 \times 10^{-2}$ \\
\hline 1 & - & 0.12 & $7.1 \times 10^{-2}$ \\
\hline $\mathrm{m}$ & - & $-6.5 \times 10^{-5}$ & $-2.6 \times 10^{-2}$ \\
\hline $\mathrm{n}$ & - & $2.6 \times 10^{-4}$ & 0.10 \\
\hline o & - & - & 0.20 \\
\hline $\mathrm{p}$ & - & - & $-5.0 \times 10^{-2}$ \\
\hline $\mathrm{q}$ & - & - & $1.1 \times 10^{-2}$ \\
\hline $\mathrm{r}$ & - & - & $7.2 \times 10^{-3}$ \\
\hline $\mathrm{s}$ & - & - & $6.4 \times 10^{-2}$ \\
\hline $\mathrm{t}$ & - & - & 0.11 \\
\hline $\mathrm{u}$ & - & - & $4.9 \times 10^{-2}$ \\
\hline $\mathrm{v}$ & - & - & 0.16 \\
\hline $\mathrm{w}$ & 2.170 & 2.6 & 0.31 \\
\hline $\mathrm{R}$ & 0.8717 & 0.9231 & 0.9829 \\
\hline $\mathrm{P}$ & 0.0007 & 0.0012 & 0.0013 \\
\hline
\end{tabular}

-, the model does not have this coefficient and relative term. 
Table 3. Mathematical models of the regression for in vitro release profiles of CUR-CNLC.

\begin{tabular}{ccc}
\hline Function & Equation & $\mathrm{R}$ \\
\hline Zero order & $\mathrm{Y}=0.05634 \mathrm{t}+0.3172$ & 0.9062 \\
First order & $\ln (1-\mathrm{Y})=-0.1425 \mathrm{t}-0.3327$ & 0.9458 \\
Higuchi & $\mathrm{Y}=0.2405 \mathrm{t}^{1 / 2}+0.1057$ & 0.9720 \\
Hixcon-Crowell & $(1-\mathrm{Y})^{1 / 3}=-0.03435 \mathrm{t}+0.8881$ & 0.9919 \\
Ritger-Peppas & $\ln \mathrm{Y}=0.4255 \ln \mathrm{t}-1.105$ & 0.9933 \\
Weibull & $\ln \ln [1 /(1-\mathrm{Y})]=0.6227 \ln -0.8824$ & \\
\hline
\end{tabular}

Y, the percentage of CUR; $t$, time point; $R$, regression coefficient. 
Table 4. The corneal permeability coefficients ( $\overline{\mathrm{X}} \pm \mathrm{SD}, \mathrm{n}=3$ ).

\begin{tabular}{cccc}
\hline Formulation & $\mathrm{P}_{\mathrm{app}} \times 10^{7}\left(\mathrm{~cm} \cdot \mathrm{s}^{-1}\right)$ & $\mathrm{J}_{\mathrm{ss}} \times 10^{6}\left(\mu \mathrm{g} \cdot \mathrm{s}^{-1} \cdot \mathrm{cm}^{-2}\right)$ & $\mathrm{HL}(\%)$ \\
\hline CUR-SOL & $3.778 \pm 0.2940$ & $1.889 \pm 0.1470$ & $81.09 \pm 0.0200$ \\
CUR-NLC & $4.693 \pm 0.5485^{*}$ & $2.346 \pm 0.2743^{*}$ & $80.21 \pm 1.063$ \\
CUR-CNLC & $4.733 \pm 0.2895^{*}$ & $2.367 \pm 0.1448^{*}$ & $80.28 \pm 0.4105$ \\
\hline
\end{tabular}

$P_{\text {app, }}$, apparent permeability coefficient; $J_{\mathrm{ss}}$, steady-state flux; HL, hydration level.

${ }^{*} P<0.05$ versus CUR-SOL. 
Table 5. Pharmacokinetic parameters of CUR in aqueous humor after topical administration in the conscious rabbits ( $\overline{\mathrm{X}} \pm \mathrm{SD}, \mathrm{n}=3)$.

\begin{tabular}{ccccc}
\hline Formulation & $\begin{array}{c}\mathrm{T}_{\max } \\
(\mathrm{min})\end{array}$ & $\begin{array}{c}\mathrm{C}_{\max } \\
\left(\mathrm{ng} \cdot \mathrm{mL}^{-1}\right)\end{array}$ & $\begin{array}{c}\text { AUC } \\
\left(\mathrm{ng} \cdot \mathrm{mL}^{-1} \cdot \mathrm{min}\right)\end{array}$ & $\begin{array}{c}\text { MRT } \\
(\mathrm{min})\end{array}$ \\
\hline CUR-SOL & $30.00 \pm 0$ & $23.11 \pm 1.915$ & $2161 \pm 55.74$ & $65.14 \pm 2.299$ \\
CUR-NLC & $103.3 \pm 11.55^{* *}$ & $27.01 \pm 4.548$ & $3334 \pm 103.1^{* *}$ & $115.3 \pm 2.312^{* *}$ \\
CUR-CNLC & $126.7 \pm 14.43^{* *}$ & $29.60 \pm 1.080^{*}$ & $5093 \pm 245.7^{* *}$ & $147.0 \pm 2.987^{* *}$ \\
\hline
\end{tabular}

$\mathrm{T}_{\max }$, time to maximal concentration; $\mathrm{C}_{\max }$, maximal concentration of CUR; AUC, area under the curve; MRT, mean residence time.

${ }^{*} P<0.05$ versus CUR-SOL; ${ }^{* *} P<0.01$ versus CUR-SOL. 


\section{Figure captions}

Figure 1. RSM showing the influence of the independent variables on OD. $\left(\mathrm{X}_{1}\right)$ concentration of lipid, $\left(\mathrm{X}_{2}\right)$ the ratio between solid lipid and liquid lipid, $\left(\mathrm{X}_{3}\right)$ concentration of surfactant, and $\left(\mathrm{X}_{4}\right)$ concentration of cationic material.

Figure 2. Physicochemical characteristics of CNLC. (a) particle size distribution, (b) zeta potential distribution.

Figure 3. DSC thermograms. (a) physical mixture of Cur-CNLC materials, (b) Cur-CNLC, (c) QACMC, (d) Myri 52, (e) Miglyol 812, (f) GMS, (g) curcumin.

Figure 4. TEM morphology of the optimal CUR-CNLC formulation.

Figure 5. In vitro release profiles of CUR from CUR-SOL, CUR-NLC and CUR-CNLC $(n=3)$.

Figure 6. Fluorescence images of rabbit eyes after administration. (a) Rh B solution, (b) Rh B CNLC. 1: ocular ROI, 2: intensity reference standard, 3: inner canthus and nasolacrimal duct region.

Figure 7. Preocular retention of various formulations.

Figure 8. CUR concentration-time profiles following a $200 \mu \mathrm{L}$ topical administration at dose of $1.0 \mathrm{mg} \cdot \mathrm{mL}^{-1}$ in aqueous humor $(\mathrm{n}=3)$. 

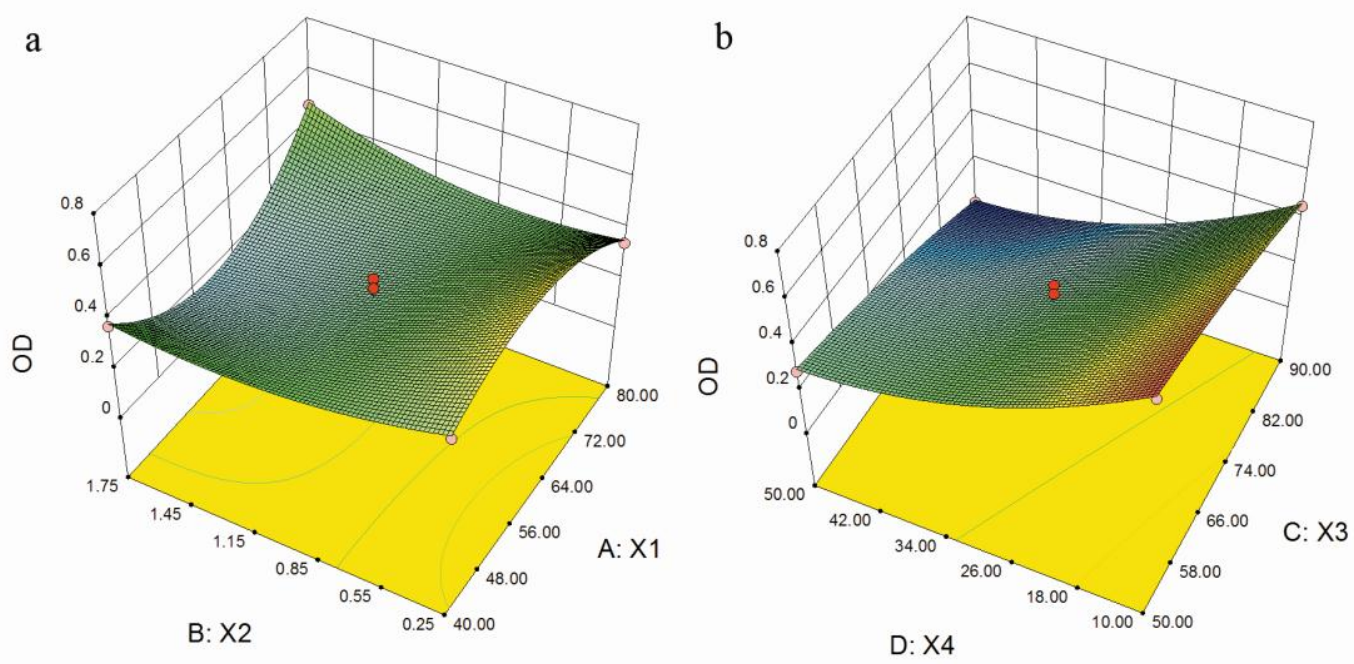
$\mathrm{a}$
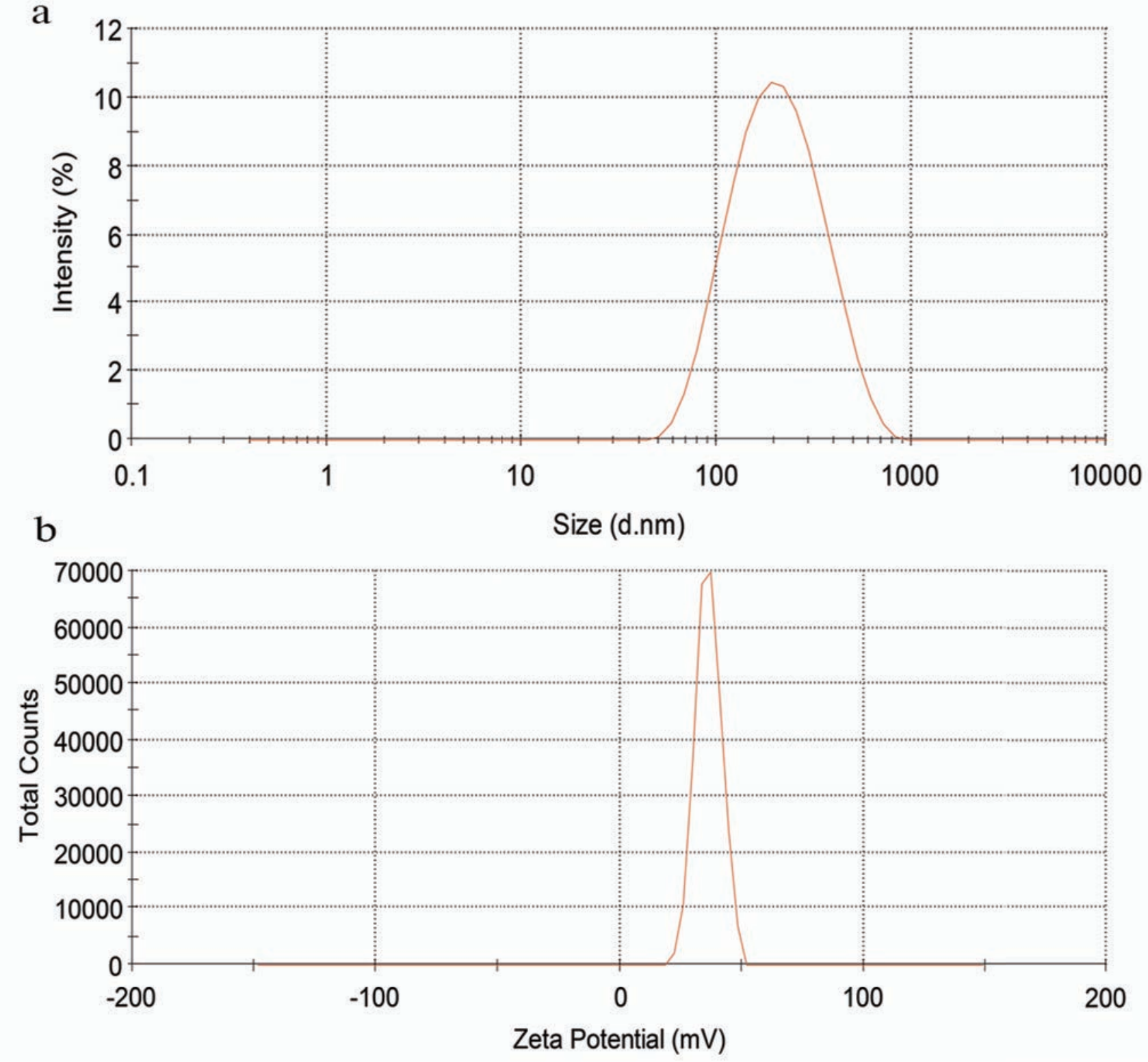
5

$-50$

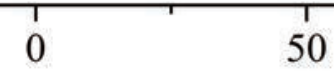

50
100

$\operatorname{Tr}\left({ }^{\circ} \mathrm{C}\right)$ 


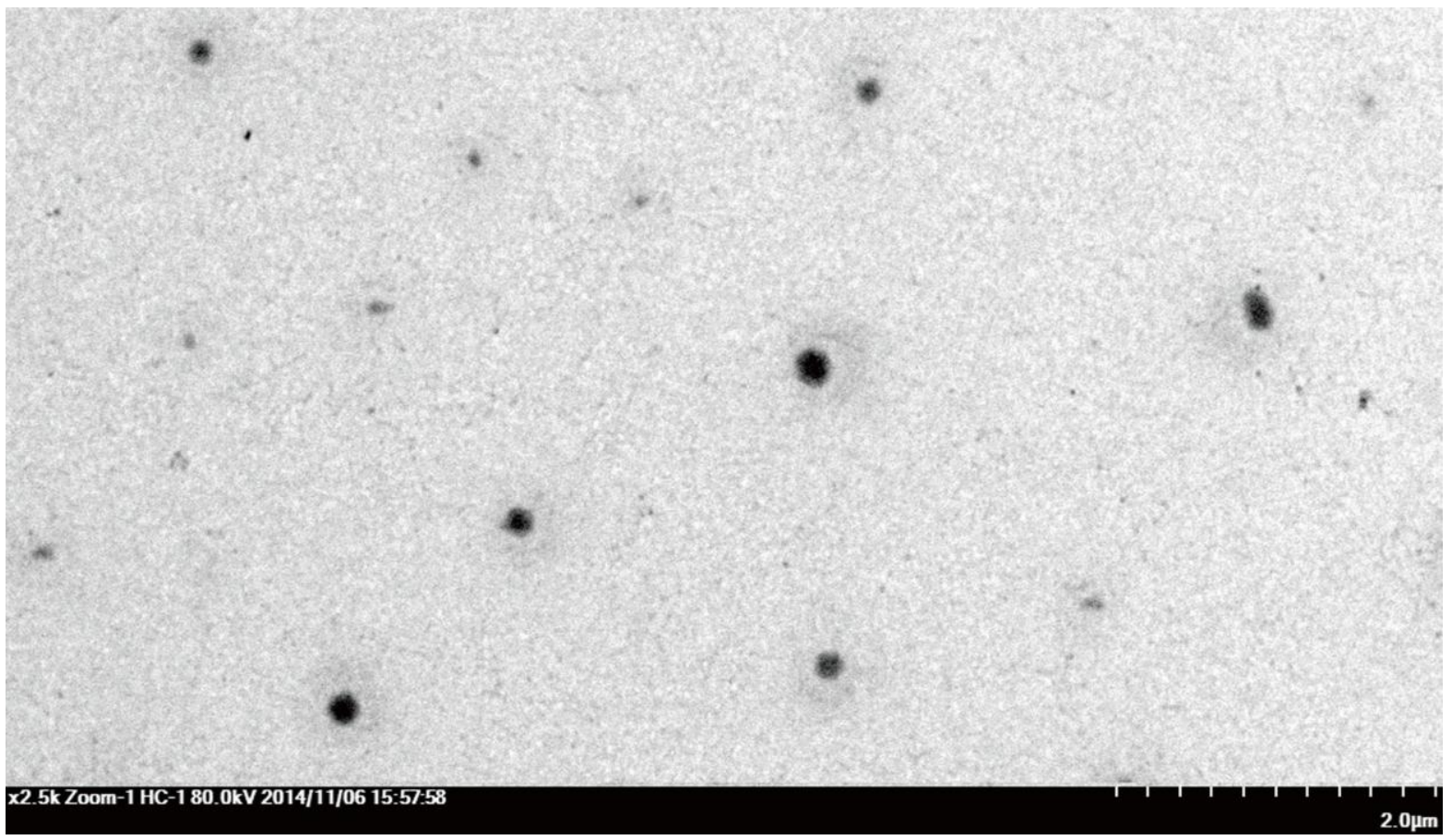




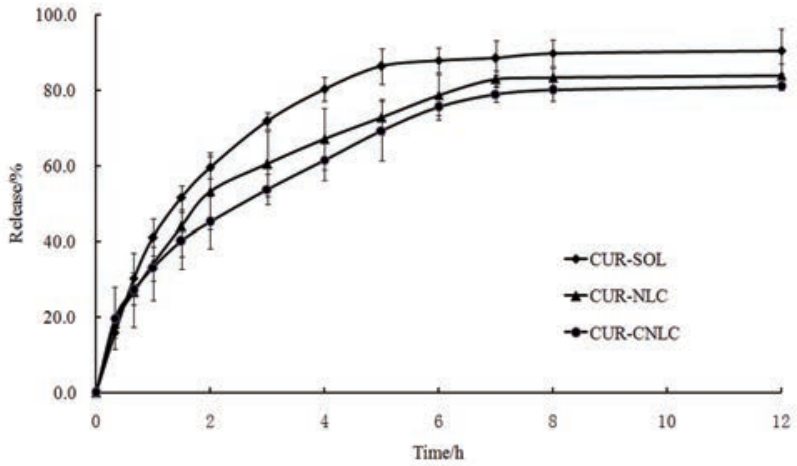




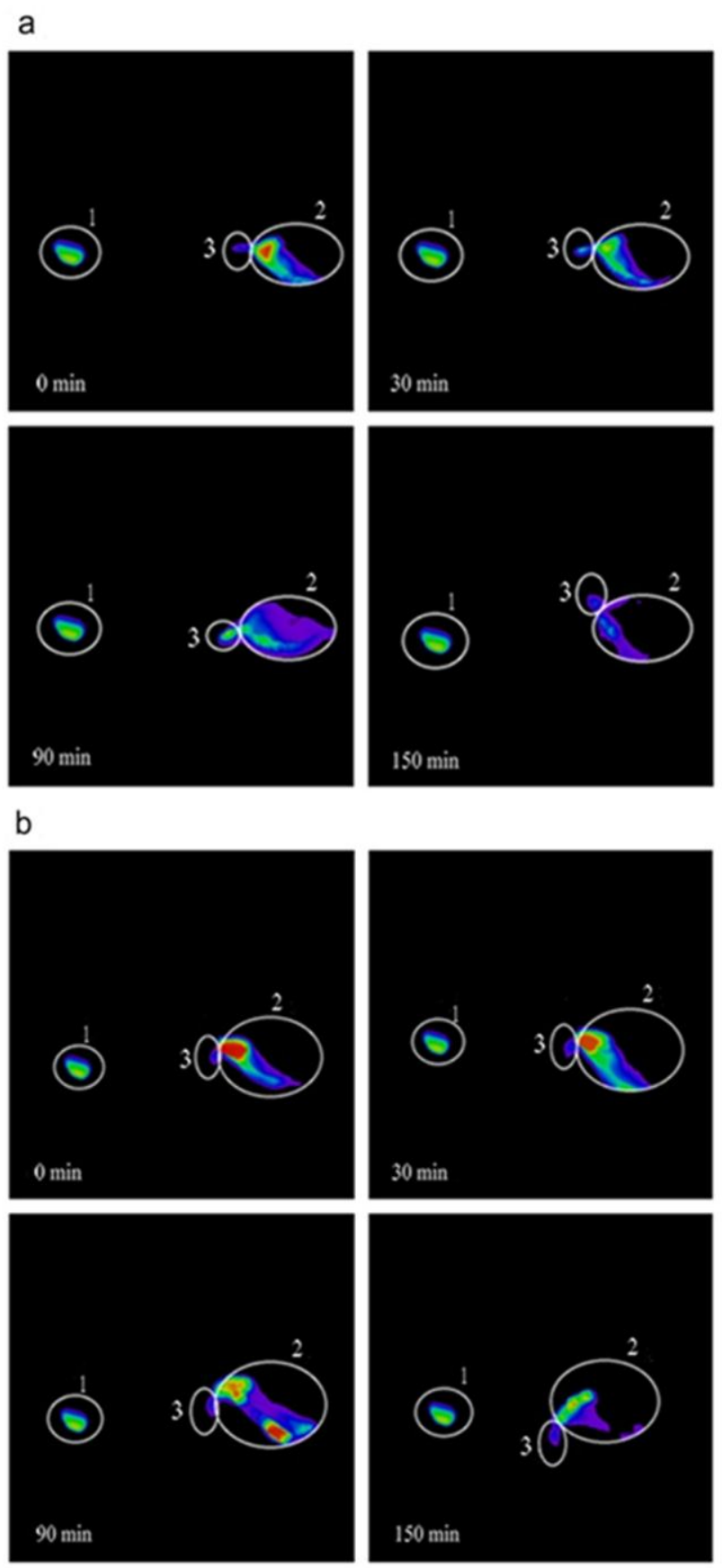




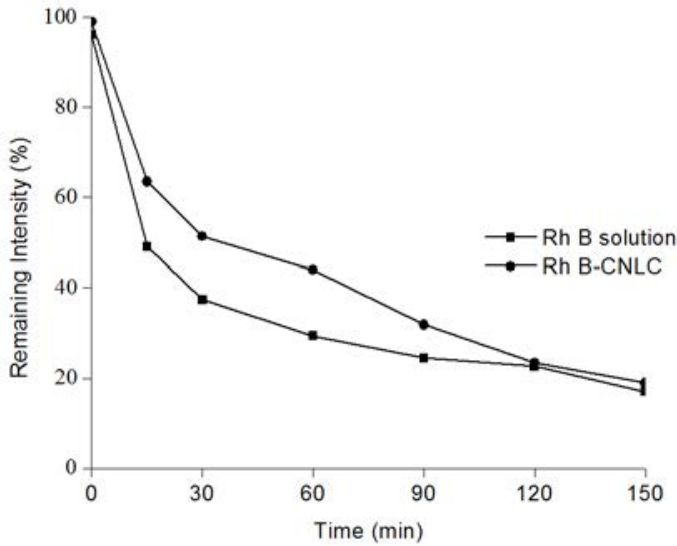




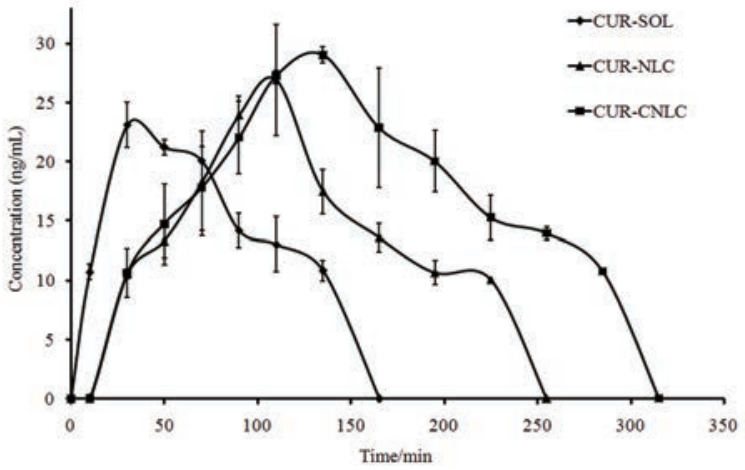



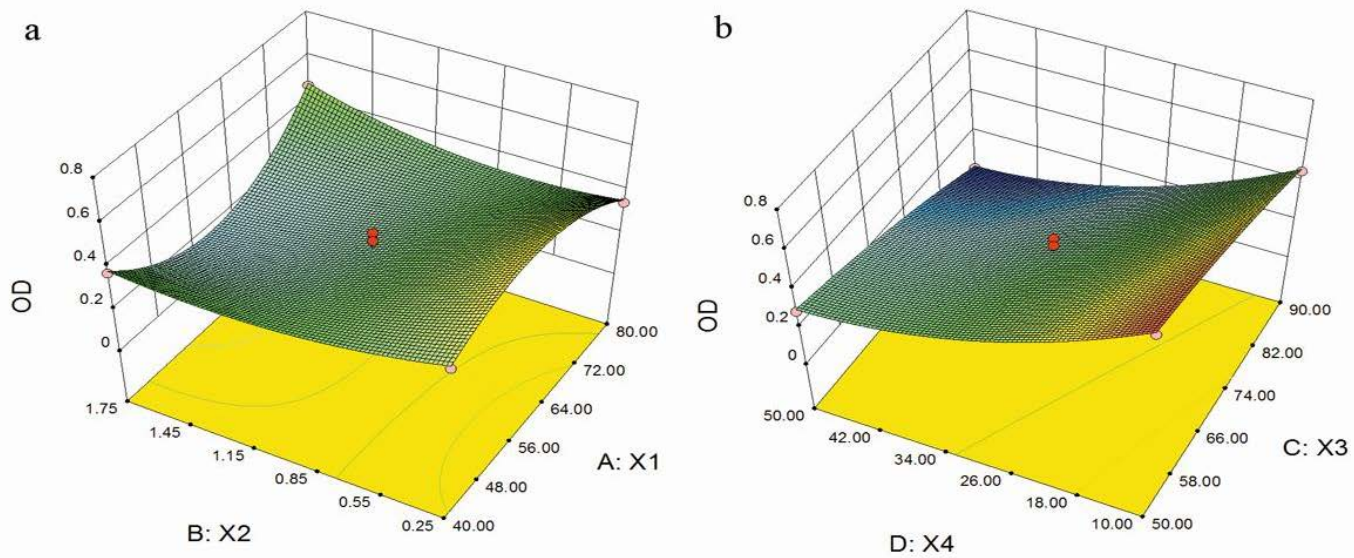